Article

\title{
Transcriptional Terminators Allow Leak-Free Chromosomal Integration of Genetic Constructs in Cyanobacteria
}

\author{
Ciarán L. Kelly®D, George M. Taylor, Aistė Šatkutè, Linda Dekker and John T. Heap * \\ Imperial College Centre for Synthetic Biology, Department of Life Sciences, Imperial College London, \\ South Kensington Campus, London SW7 2AZ, UK \\ * Correspondence: j.heap@imperial.ac.uk
}

Received: 2 July 2019; Accepted: 12 August 2019; Published: 16 August 2019

\begin{abstract}
Cyanobacteria are promising candidates for sustainable bioproduction of chemicals from sunlight and carbon dioxide. However, the genetics and metabolism of cyanobacteria are less well understood than those of model heterotrophic organisms, and the suite of well-characterised cyanobacterial genetic tools and parts is less mature and complete. Transcriptional terminators use specific RNA structures to halt transcription and are routinely used in both natural and recombinant contexts to achieve independent control of gene expression and to 'insulate' genes and operons from one another. Insulating gene expression can be particularly important when heterologous or synthetic genetic constructs are inserted at genomic locations where transcriptional read-through from chromosomal promoters occurs, resulting in poor control of expression of the introduced genes. To date, few terminators have been described and characterised in cyanobacteria. In this work, nineteen heterologous, synthetic or putative native Rho-independent (intrinsic) terminators were tested in the model freshwater cyanobacterium, Synechocystis sp. PCC 6803, from which eleven strong terminators were identified. A subset of these strong terminators was then used to successfully insulate a chromosomally-integrated, rhamnose-inducible rhaBAD expression system from hypothesised 'read-through' from a neighbouring chromosomal promoter, resulting in greatly improved inducible control. The addition of validated strong terminators to the cyanobacterial toolkit will allow improved independent control of introduced genes.
\end{abstract}

Keywords: transcriptional terminators; cyanobacteria; Synechocystis; inducible expression; synthetic biology

\section{Introduction}

Cyanobacteria are important photosynthetic model organisms and potential photosynthetic microbial factories. The ability to reliably engineer photosynthetic organisms could enable the sustainable, carbon-neutral, light-driven conversion of carbon dioxide to valuable products using energy from sunlight. Predictable engineering of cyanobacteria remains challenging for many reasons, one of which is a shortage of well-characterised genetic parts such as promoters, ribosome-binding sites and transcriptional terminators [1,2].

We recently described the successful introduction of a rhamnose-inducible rhaBAD expression system into the cyanobacterium Synechocystis sp. PCC 6803 (Synechocystis hereafter). This expression system has superior properties to many other previously reported inducible promoter systems, including low basal expression in the absence of inducer, a photostable and non-toxic inducer and a linear response to inducer concentration [3]. However, when introduced into the Synechocystis chromosome adjacent to the $n d h B$ gene, we observed a non-zero basal level of expression, which 
we attributed to transcriptional read-through from promoter(s) neighbouring the integration site used. Chromosomal integration is important for the stability of expression constructs, but in cases where extremely low or zero basal expression is required, transcriptional read-through can result in unpredictable gene expression, growth defects, toxicity and genetic instability.

Transcriptional terminators can be used to achieve independent control of gene expression and isolate or 'insulate' genes and operons from one another and from neighbouring elements when chromosomally integrated. In contrast to Escherichia coli, in which the use and characterisation of transcriptional terminators is routine, evaluation of the function of terminators in Synechocystis has been largely ignored to date. There are two main types of terminators: Rho-dependent terminators and Rho-independent or 'intrinsic' terminators. Rho-dependent termination requires a homohexameric Rho protein that unwinds the RNA-DNA hybrid, thus halting elongation of nascent RNA strands. No homologues of Rho have been identified in cyanobacterial genomes to date [4]. Rho-independent termination results from the formation of a hairpin-loop secondary structure in the nascent RNA strand, causing dissociation of the transcription elongation complex (comprising RNA polymerase, double-stranded DNA and nascent RNA). Termination is intrinsic to the nucleotide sequence of the RNA strand itself and composed of an adenosine-rich tract (A-tract) located upstream of a hairpin loop consisting of a GC-rich stem region (4-18 bp) and loop nucleotides (3-5 bp), followed by a highly conserved uracil-rich tract (U-tract; 6-8 bp). Transcription of the U-tract transiently pauses the elongation complex, allowing formation of the hairpin loop. This destabilises the complex, resulting in DNA:RNA hybrid shearing, termination of elongation and release of the partial transcript. Interestingly, an analysis of RNA-folding energetics near stop codons in Synechocystis suggested a lack of RNA hairpin-loop formation at these sites, implying Rho-independent termination is not prevalent in this cyanobacterium [4,5]. A comprehensive analysis of terminators in Synechocystis has not been carried out to date.

In this work we screened nineteen Rho-independent, transcriptional terminator sequences in Synechocystis. A subset of the best performing terminators was then tested for their ability to insulate a previously described [3] rhamnose-inducible yellow fluorescent protein (YFP) reporter cassette from likely transcriptional read-through at the site of chromosomal integration. Introduction of any of the chosen terminators resulted in a basal level of YFP production that was indistinguishable from Synechocystis cells lacking the YFP expression cassette entirely, confirming successful insulation of chromosomally integrated constructs.

\section{Materials and Methods}

\subsection{Bacterial Strains and Growth Conditions}

E. coli strain DH10B was used for all plasmid construction and propagation and the wild-type K12 strain MG1655 used for terminator-verification assays. Synechocystis sp. PCC 6803 WT-G (the glucose-tolerant derivative of the wild type, originally a kind gift from the laboratory of Peter Nixon at Imperial College London, London, U.K.) was used for all cyanobacterial experiments. E. coli was cultured in LB media at $37^{\circ} \mathrm{C}$ with shaking at $240 \mathrm{rpm}$ and Synechocystis was cultured in TES-buffered (pH 8.2) BG11 media [6] with $5 \mathrm{mM}$ glucose (mixotrophic growth) or without glucose (photoautotrophic growth) at $30^{\circ} \mathrm{C}$ with shaking at $150 \mathrm{rpm}$, supplemented with $30 \mu \mathrm{g} \mathrm{mL}^{-1} \mathrm{kanamycin}$ where required. Synechocystis was grown in constant white light at $50 \mu \mathrm{mol} \mathrm{m} \mathrm{m}^{-2}$.

\subsection{Plasmid Construction}

A table of all plasmids and oligonucleotides (Table S1) is provided in the Supplementary Information. Terminators were introduced as follows. Each terminator sequence was split in two at the hairpin-loop sequence and each part was included at the $5^{\prime}$ end of oligonucleotides that were then used to amplify pCK306. PCR fragments were then ligated by blunt-end ligation using the 
New England Biolabs site-directed mutagenesis kit (New Englands Biolabs, Ipswich, MA, USA) and sequence verified.

\subsection{Strain Construction}

Synechocystis transformants were constructed as previously described [3] and full segregation confirmed by PCR.

\subsection{Assays}

Synechocystis transformants were cultured without glucose (photoautotrophic growth) for $24 \mathrm{~h}$. These pre-cultures were then used to inoculate $5 \mathrm{~mL}$ of BG11 media to an OD (measured at $750 \mathrm{~nm}$ ) of 0.1 , in the presence of $0.6 \mathrm{mg} / \mathrm{mL}$ L-rhamnose. The YFP fluorescence of samples at various time points (indicated in text) was then measured as previously described [3] by flow cytometry using an Attune NxT Flow Cytometer (ThermoFisher, Waltham, MA, USA). Cells were gated using forward and side scatter, and YFP fluorescence (excitation and emission wavelengths: $488 \mathrm{~nm}$ and $525 \mathrm{~nm}$ [with $20 \mathrm{~nm}$ bandwidth] respectively) was measured. Histograms of fluorescence intensity were plotted, and mean statistics extracted. E. coli transformants were cultured in LB media with or without $0.6 \mathrm{mg} / \mathrm{mL}$ L-rhamnose for $6 \mathrm{~h}$ and YFP fluorescence assayed as previously described [7] by flow cytometry. Error bars shown represent the standard deviation of three independent biological repeats. Statistical significance was determined using a one-way ANOVA, followed by a Tukey's multiple comparison test assuming unequal variance.

\section{Results}

\subsection{Screening Rho-independent Terminators}

Rho-independent terminators were identified from the literature including twelve strong, natural terminators from E. coli [8] (two of which were taken from the Biobricks registry of biological parts and used previously in cyanobacteria but not tested for efficacy $[2,9,10])$, four synthetic terminators that showed excellent transcriptional termination in E. coli [8], one putative cyanobacterial terminator [11] and T21 and M13 bacteriophage terminators [12,13] (Table 1).

In order to screen the nineteen terminators for transcriptional termination activity in Synechocystis, they were inserted immediately downstream of the transcriptional start site (TSS+1) of the E. coli rhaBAD promoter and upstream of the ribosome-binding site (RBS) in front of the YFP-encoding gene on plasmid pCK306 [3] (Figure 1A). This Synechocystis-E. coli shuttle plasmid both replicates stably in E. coli and integrates by homologous recombination into the Synechocystis genome at a site adjacent to the $n d h B$ gene. The rhaBAD promoter allows a linear induction of YFP production with respect to the concentration of the inducer (L-rhamnose) added to the growth medium [3]. The resulting plasmids pAS001, pAS002, pAS004-pAS020 were verified by sequencing and initially tested in two commonly used E. coli strains, DH10B and MG1655. E. coli transformants were grown in rich media with or without $0.6 \mathrm{mg} / \mathrm{mL}$ L-rhamnose at $37^{\circ} \mathrm{C}$ and fluorescence was measured after $6 \mathrm{~h}$ by flow cytometry, as previously described [3]. YFP production was abolished in all transformants containing the integrated terminator constructs compared to the positive control pCK306 transformants (highly statistically-significant difference; $p$ value $<0.0001$ ) (Figure S1). No statistically-significant difference in fluorescence was observed between pAS001, pAS002, and pAS004-pAS020 transformants grown with or without inducer. Indeed, no statistically-significant difference could be observed between the basal fluorescence of pAS001, pAS002, and pAS004-pAS020 transformants in the presence of inducer and negative control pCK324 transformants, which lack the rhaBAD promoter entirely. Subtle differences were observed between the two E. coli strains, for example statistically-significant YFP production with inducer was observed with plasmids pAS017 and pAS020 in MG1655 but not in DH10B ( $p$ values of $<0.0001$ and $<0.05$ respectively). 
Table 1. Terminators used in this study.

\begin{tabular}{|c|c|c|c|c|c|c|}
\hline Terminator & Plasmid & Length (bp) & Sequence $\left(5^{\prime}-3^{\prime}\right)$ & $\Delta \mathrm{G}(\mathrm{kcal} / \mathrm{mol})$ & Origin & References \\
\hline ECK120029600 & pAS001 & 90 & $\begin{array}{l}\text { TTCAGCCAAAAAACTTAAGACCGCCGGTCTTGTCCACTACCTTGC } \\
\text { AGTAATGCGGTGGACAGGATCGGCGGTTTTCTTTTCTCTTCTCAA }\end{array}$ & -42.00 & E. coli $\mathrm{K} 12$ & [8] \\
\hline $\begin{array}{l}\text { ECK120033737; thrL } \\
\text { attenuator }\end{array}$ & pAS002 & 57 & GGAAACACAGAAAAAAGCCCGCACCTGACAGTGCGGGCTTTTTTTTTTCGACCAAAGG & -25.00 & E. coli $\mathrm{K} 12$ & {$[8,12]$} \\
\hline ECK120034435 & pAS004; pCK351 & 57 & CTCGGTACCAAATTCCAGAAAAGAGACGCTGAAAAGCGTCTTTTTTCGTTTTGGTCC & -27.90 & E. coli $\mathrm{K} 12$ & [8] \\
\hline L3S2P21 & pAS005 & 61 & CTCGGTACCAAATTCCAGAAAAGAGGCCTCCCGAAAGGGGGGCCTTTTTTCGTTTTGGTCC & -37.90 & Synthetic & [8] \\
\hline L3S2P56 & pAS006 & 57 & CTCGGTACCAAATTTTCGAAAAAAGACGCTGAAAAGCGTCTTTTTTCGTTTTGGTCC & -28.80 & Synthetic & [8] \\
\hline L3S2P51 & pAS007 & 57 & CTCGGTACCAAAAAAAAAAAAAAAGACGCTGAAAAGCGTCTTTTTTCGTTTTGGTCC & -24.90 & Synthetic & [8] \\
\hline L3S1P56 & pAS008 & 52 & TTTTCGAAAAAAGGCCTCCCAAATCGGGGGGCCTTTTTTATTGATAACAAAA & -23.40 & Synthetic & [8] \\
\hline $\begin{array}{l}\text { Bba_B0015; } \\
\text { rrnB terminator }\end{array}$ & pAS009 & 130 & $\begin{array}{l}\text { CCAGGCATCAAATAAAACGAAAGGCTCAGTCGAAAGACTGGGCCTTTCGTTTTATCTGTTGTTT } \\
\text { GTCGGTGAACGCTCTCTACTAGAGTCACACTGGCTCACCTTCGGGTGGGCCTTTCTGCGTTTATA }\end{array}$ & -72.10 & E. coli $\mathrm{K} 12$ & [2] \\
\hline ECK120035133 & pAS010 & 43 & ACTGATTTTTAAGGCGACTGATGAGTCGCCTTTTTTTTGTCT & -15.40 & E. coli $\mathrm{K} 12$ & [8] \\
\hline ECK120017009 & pAS011 & 44 & GATCTAACTAAAAAGGCCGCTCTGCGGCCTTTTTTCTTTTCACT & -16.20 & E. coli $\mathrm{K} 12$ & [8] \\
\hline ECK120015170 & pAS012; pCK353 & 47 & ACAATTTTCGAAAAAACCCGCTTCGGCGGGTTTTTTTATAGCTAAAA & -20.10 & E. coli $\mathrm{K} 12$ & [8] \\
\hline ECK120033736 & pASO13 & 53 & AACGCATGAGAAAGCCCCCGGAAGATCACCTTCCGGGGGCTTTTTTATTGCGC & -37.80 & E. coli $\mathrm{K} 12$ & [8] \\
\hline ECK120010799 & pAS014; pCK354 & 60 & GTTATGAGTCAGGAAAAAAGGCGACAGAGTAATCTGTCGCCTTTTTTCTTTGCTTGCTTT & -33.60 & E. coli $\mathrm{K} 12$ & [8] \\
\hline $\begin{array}{l}\text { BBa_B0010; } \\
\text { rrnB terminator }\end{array}$ & pAS015 & 80 & $\begin{array}{l}\text { CCAGGCATCAAATAAAACGAAAGGCTCAGTCGAAAGA } \\
\text { CTGGGCCTTTCGTTTTATCTGTTGTTTGTCGGTGAACGCTCTC }\end{array}$ & -42.90 & E. coli $\mathrm{K} 12$ & [9] \\
\hline$\Omega g r o E L$ & pAS016 & 89 & $\begin{array}{l}\text { GGTTTAGTAGACCGACTACCACTTTTCTCATAAAATCCCA } \\
\text { GGGAGGTTTCGGCCTCCCTTTTTTTCACTTGCTAAGCTCTCTTTCGTTT }\end{array}$ & -20.80 & $\begin{array}{l}\text { Synechocystis sp. } \\
\text { PCC } 6803\end{array}$ & [11] \\
\hline T21 & pAS017 & 74 & $\begin{array}{l}\text { ATTGAGCAAGTAGCAACACTATTCGCATAAGCTGCC } \\
\text { GTTAGTGACTCTTAAGTTGCAACGGTGGCTTTTTTTAT }\end{array}$ & -25.40 & $\begin{array}{l}\text { Bacteriophage } \\
\text { T21 }\end{array}$ & [13] \\
\hline M13 Central & pAS018 & 85 & $\begin{array}{l}\text { AAAGCAAGCTGATAAACCGATACAATTAAAGGCTCCTTTT } \\
\text { GGAGCCTTTTTTTTTGGAGATTTTCAACATGAAAAAATTATTATT }\end{array}$ & -18.60 & $\begin{array}{l}\text { Bacteriophage } \\
\text { M13 }\end{array}$ & [13] \\
\hline$i l v B N$ terminator & pAS019; pCK355 & 36 & AAGACCCCCGCACCGAAAGGTCCGGGGGTTTTTTTT & -24.40 & E. coli $\mathrm{K} 12$ & {$[8,13]$} \\
\hline ECK120010793 & pAS020 & 34 & TACGTAAAAACCCGCTTCGGCGGGTTTTTACTTT & -24.40 & E. coli $\mathrm{K} 12$ & {$[8,13]$} \\
\hline
\end{tabular}


A

pCK306 integration vector
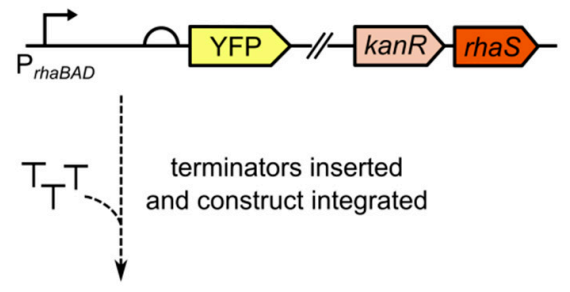

chromosomally-integrated pAS001-020

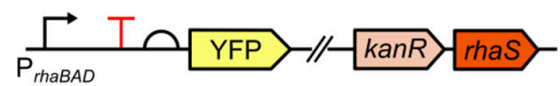

B

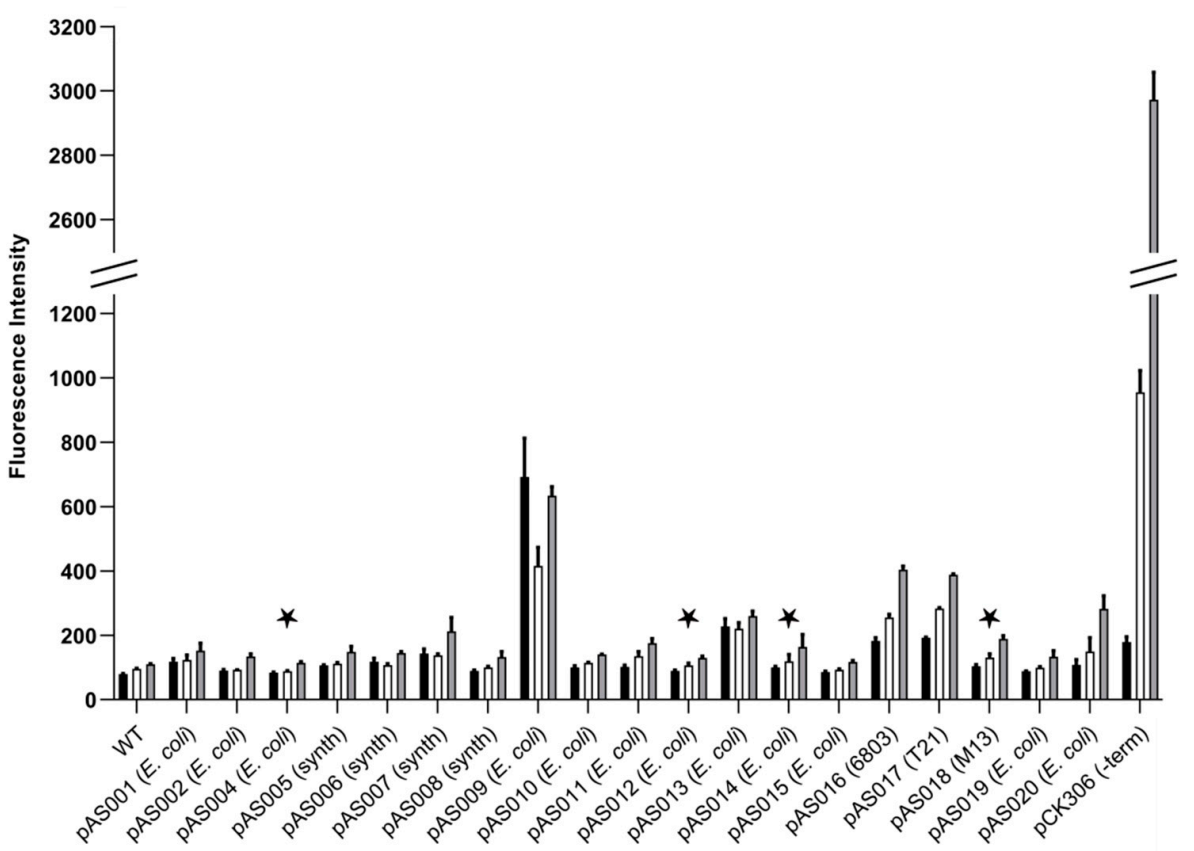

Figure 1. Screening of terminators in Synechocystis. (A) Overview showing insertion of terminators into plasmid pCK306 between the rhaBAD promoter and the ribosome-binding site (RBS) of the yellow fluorescent protein (YFP)-encoding gene. The resulting terminator constructs pAS001, pAS002, pAS004-020 were integrated into the Synechocystis genome. (B) Synechocystis transformants containing the integrated terminator constructs were cultured in BG11 media supplemented with kanamycin and $0.6 \mathrm{mg} / \mathrm{mL}$ L-rhamnose in photoautotrophic conditions in constant light. Wild-type Synechocystis cells (WT) lacking YFP entirely and cells containing the YFP-encoding cassette of the parent plasmid pCK306 (no terminator between rhaBAD promoter and RBS of YFP-encoding gene) were used as controls. The fluorescence intensity (arbitrary units) of 10,000 cells from each culture was measured by flow cytometry at $0 \mathrm{~h}$ (immediately after the culture was inoculated), $48 \mathrm{~h}$ and $96 \mathrm{~h}$ (black, white and grey bars respectively). Error bars shown are the standard deviation of the mean for three independent biological replicates. Key for SBOL glyphs used in figure: right-angled arrow represents a promoter; T represents a terminator; semi-circle represents a ribosome-binding site (RBS); coloured blocks represent coding sequences or genes. Origin of each terminator in brackets after plasmid name: E. coli, synth (synthetic), 6803 (Synechocystis sp. PCC 6803), T21 (bacteriophage T21), M13 (bacteriophage M13). Stars above terminators refer to the four terminators that were used in the chromosomal insulation experiments (see Figure 2).

Following characterisation in E. coli, the terminator-containing plasmids pAS001, pAS002, pAS004-pAS020 were integrated into the Synechocystis genome, with full segregation confirmed by PCR. The resulting transformants were cultured photoautotrophically, at $30{ }^{\circ} \mathrm{C}$ under constant light in BG11 growth medium supplemented with kanamycin and $0.6 \mathrm{mg} / \mathrm{mL}$ L-rhamnose. YFP fluorescence was measured by flow cytometry after 0,48 and 96 h (linear phase of growth, as shown in Figure S3) 
and compared to wild-type (no YFP) Synechocystis cells and pCK306 (parent plasmid with no terminator inserted) transformants as controls (Figure 1). All pAS001, pAS002, pAS004-pAS020 transformants showed lower fluorescence than pCK306 transformants at $48 \mathrm{~h}$ and $96 \mathrm{~h}$ (highly statistically-significant difference; $p$ value $<0.0001$ ), indicating YFP production is reduced by the presence of any of these terminators. No significant difference in fluorescence was observed between wild-type cells and fifteen of the terminator-plasmid transformants at $48 \mathrm{~h}$, with pAS009, pAS013, pAS016 and pAS017 showing significant differences ( $p$ values of $<0.0001$ for all). At $96 \mathrm{~h}$, no significant difference in fluorescence was observed between wild-type cells and twelve of the terminator-plasmid transformants, with pAS007, pAS009, pAS013, pAS016, pAS017, pAS018 and pAS020 transformants showing significant differences (respective $p$ values of $<0.001,<0.0001,<0.0001,<0.0001,<0.0001,<0.05$ and $<0.0001$ ). These data show that twelve of the nineteen terminators tested in Synechocystis are strong transcriptional terminators, as they lead to YFP levels that are similar to those found in cells lacking YFP altogether. This conclusion is further supported by analysis of each transformant over the three timepoints, where no significant change in fluorescence was observed across the entire time course for eleven of these terminators. A significant difference $(p$ value $<0.005)$ was only observed for pAS011 between $0 \mathrm{~h}$ and $96 \mathrm{~h}$.

\subsection{Strong Terminators Insulate Expression Constructs Integrated in the Synechocystis Chromosome from Transcriptional Read-Through}

Four of the eleven strong terminator sequences were used to attempt to insulate integrated expression constructs from chromosomal read-through. These were terminators ECK120034435, ECK120015170, ECK120010799 and the E. coli ilvBN terminator, as originally screened using plasmids pAS004, pAS012, pAS014 and pAS019, respectively. Each of these terminators was introduced immediately upstream of the rhaBAD promoter of pCK306 (Figure 2A), resulting in plasmids pCK351, pCK353, pCK354 and pCK355, respectively. The four constructs were integrated into the Synechocystis genome at the locus adjacent to $n d h B$ and full segregation was confirmed by PCR. Synechocystis transformants containing integrated pCK351, pCK353, pCK354 and pCK355 constructs were cultured at $30^{\circ} \mathrm{C}$ under constant light in BG11 growth medium supplemented with kanamycin, with glucose (mixotrophic) or without glucose (photoautotrophic) and with or without $0.6 \mathrm{mg} / \mathrm{mL}$ L-rhamnose. YFP fluorescence was measured by flow cytometry after $0 \mathrm{~h}, 96 \mathrm{~h}$ and $192 \mathrm{~h}$ (linear phase of growth, as shown in Figure S4) and compared to wild-type (no YFP) Synechocystis cells and pCK306 (parent plasmid with no terminator inserted) transformants as controls. In the absence of inducer, the introduction of each of the four terminators reduced the YFP fluorescence of these cells by 1.7-2.1 times compared to pCK306 fluorescence (highly statistically-significant differences; $p$ values of $<0.0001$ ) in both photoautotrophic and mixotrophic growth conditions when measured at $96 \mathrm{~h}$ (Figure 2B,C). This resulted in very low fluorescence levels that were indistinguishable from wild-type cells lacking YFP entirely (no statistically-significant difference). The same pattern of results was observed at the later $192 \mathrm{~h}$ timepoint (Figure S2B,C). These data indicate that these four terminators are indeed effective at preventing transcriptional read-through from a chromosomal promoter and basal transcription has been reduced to undetectable levels. The four Synechocystis transformants containing YFP expression constructs insulated by upstream terminators were next tested to ensure inducible YFP production was still possible. Under photoautotrophic growth conditions, no statistically-significant difference in successfully induced fluorescence was observed between each of the four transformants and the parent control pCK306 transformant at either 96 h (Figure 2D) or $192 \mathrm{~h}$ (Figure S2D). Under mixotrophic growth conditions, no significant difference in successfully induced fluorescence was observed at either $96 \mathrm{~h}$ or $192 \mathrm{~h}$, when pCK351 and pCK353 transformants were compared to the control transformant pCK306 (Figure 2E). A lower level of YFP was observed in transformants containing pCK354 and pCK355 constructs at the $96 \mathrm{~h}$ timepoint compared with pCK306 transformants (highly statistically-significant difference $p$ value < 0.0001) (Figure 2E). This difference had disappeared however at the later $192 \mathrm{~h}$ timepoint (Figure S2E). 
A

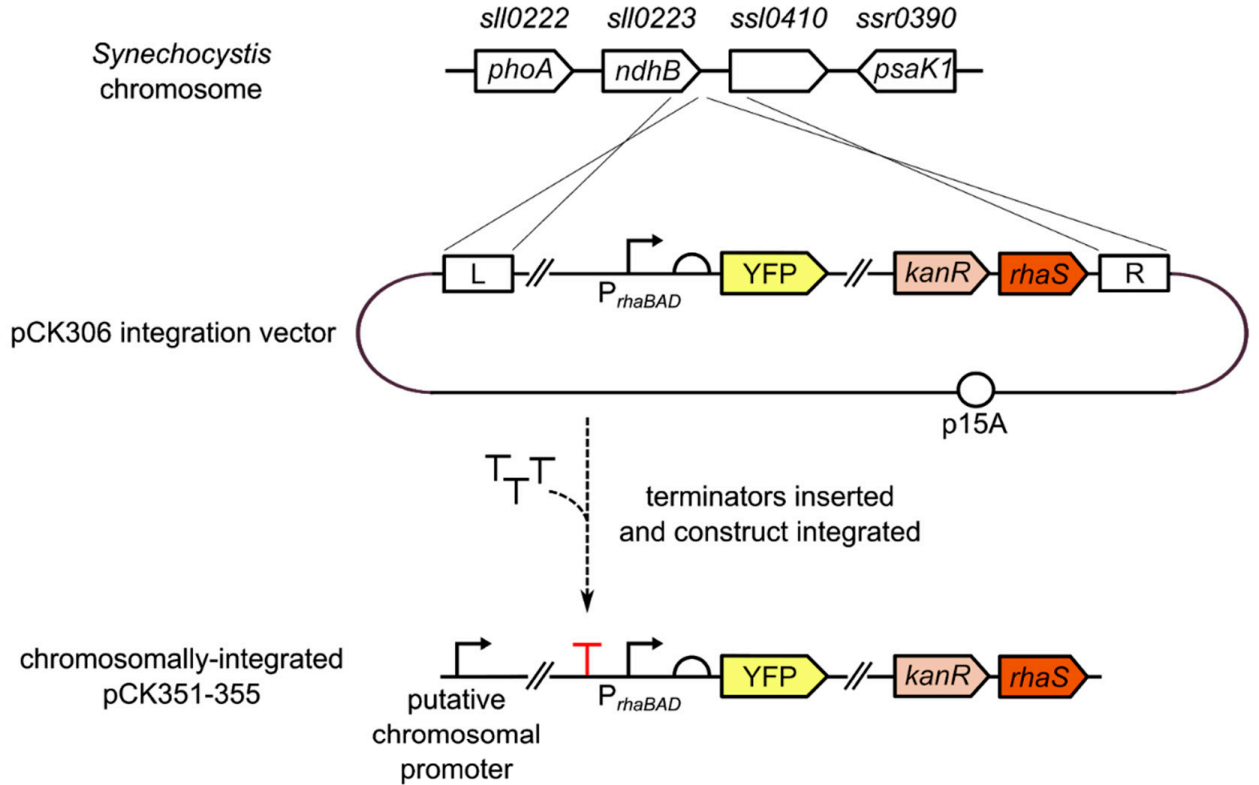

B
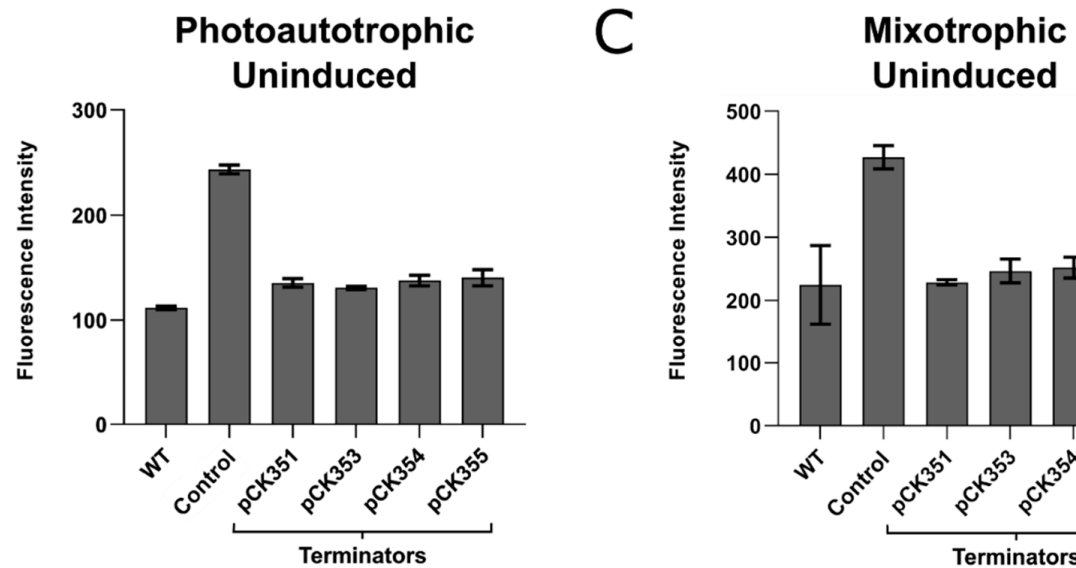

D
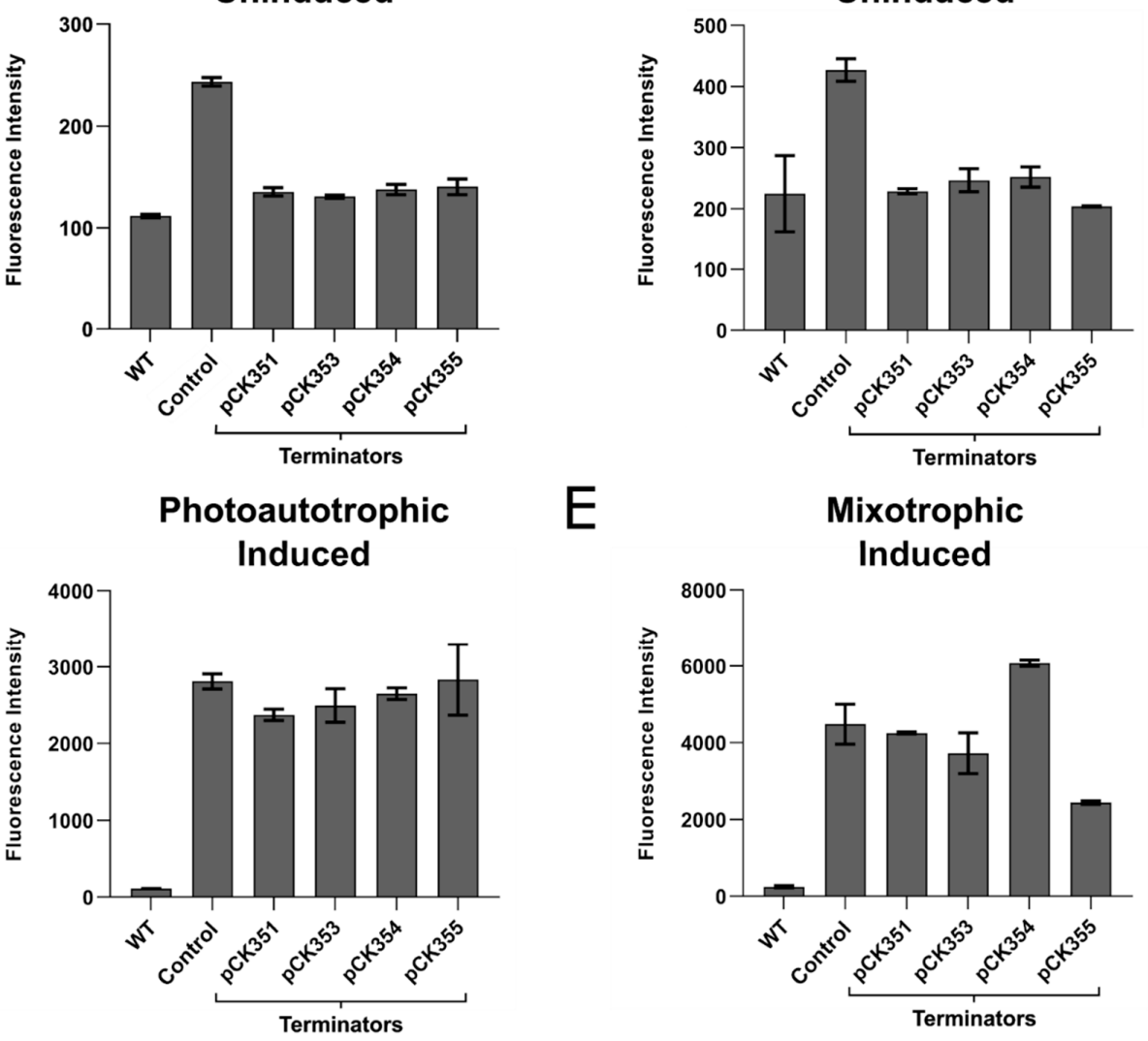

E

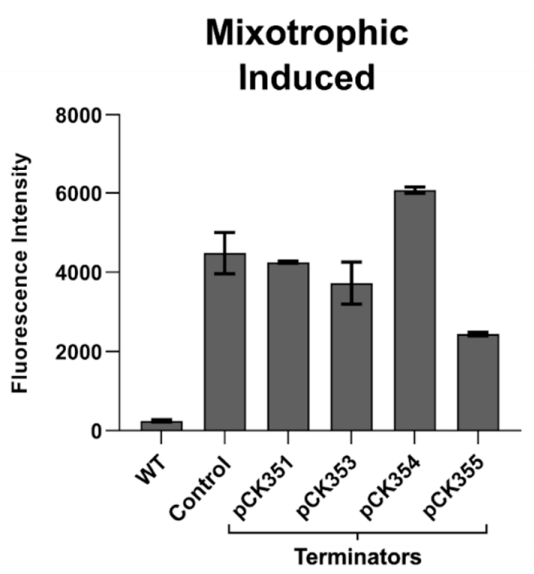

Figure 2. The effect of terminators inserted upstream of a rhamnose-inducible YFP expression construct inserted in the Synechocystis chromosome after $96 \mathrm{~h}$. (A) Detail showing the insertion of terminators into integration plasmid pCK306 upstream of the rhaBAD promoter. The resulting constructs pCK351, pCK353, pCK354 and pCK355 were integrated into the Synechocystis genome adjacent to the $n d h B$ gene. (B) To test for transcriptional insulation from chromosomal promoters after integration, Synechocystis transformants containing the integrated terminator constructs were cultured in BG11 media supplemented with kanamycin and no L-rhamnose, in photoautotrophic conditions and 
constant light. Wild-type Synechocystis cells (WT) lacking YFP entirely and cells containing pCK306 (no terminator inserted upstream of rhaBAD promoter) were used as controls. The fluorescence intensity (arbitrary units) of 10,000 cells from each culture was measured by flow cytometry at $96 \mathrm{~h}$. (C) Equivalent experiment to (B) but strains cultured in BG11 supplemented with $5 \mathrm{mM}$ D-glucose (mixotrophic growth). (D) The same strains of Synechocystis were cultured in BG11 media supplemented kanamycin and L-rhamnose to a final concentration of $0.6 \mathrm{mg} / \mathrm{mL}$ in photoautotrophic conditions and constant light. The fluorescence intensity (arbitrary units) of 10,000 cells was measured after $96 \mathrm{~h}$ using flow cytometry. (E) Equivalent experiment to (D) but strains cultured in BG11 supplemented with $5 \mathrm{mM}$ D-glucose (mixotrophic growth). Error bars shown represent the standard deviation of the mean of three independent biological replicates. Key for SBOL glyphs used in figure: right-angled arrow represents a promoter; $\mathrm{T}$ represents a terminator; semi-circle represents a ribosome-binding site (RBS); coloured blocks represent coding sequences or genes.

\section{Discussion}

In this study, nineteen Rho-independent terminators were evaluated in Synechocystis. This is important, as although a small number of E. coli-derived terminator sequences have previously been used in genetic constructs in cyanobacteria $[2,9,11]$, no characterisation of their efficacy had been performed until now. The subunits of RNA polymerase differ between E. coli and Synechocystis and it has been reported that differences in RNA polymerase can lead to different termination efficiencies of terminators in different organisms [12]. Therefore, it was not obvious that all strong E. coli terminators would work well in Synechocystis. During preparation of this manuscript the characterisation of seven putative terminators found in the Synechocystis genome was reported [14], with moderate termination efficiencies observed. In our study, non-native and synthetic sequences were included, and twelve strong Rho-independent terminators identified.

We demonstrated that four of the strongest terminators allow complete insulation of a chromosomally integrated expression construct from transcriptional read-through resulting from genomic promoter(s) neighbouring the site of integration, presumably promoters of one or more of the sll0222 (phoA), sll0223 $(n d h B)$ or ssl0410 genes [15]. In particular, we used strong terminators to improve the cyanobacterial rhamnose-inducible rhaBAD expression system previously reported [3,7], reducing basal transcription to undetectable levels. Importantly, this terminator insulation did not affect the inducible control of the expression system. The terminators characterised in this work are a valuable addition to the synthetic biology toolbox for engineering of cyanobacteria for fundamental and biotechnology purposes, allowing the insulation and independent control of individual expression units encoding separate components of a metabolic pathway or system. We recently used some of the terminators studied here in the combinatorial construction and optimisation of libraries of metabolic pathways in Synechocystis using the organism-independent Start-Stop Assembly system [16], as will be described in a forthcoming publication (Taylor and Heap, unpublished results). The improved and fully insulated rhamnose-inducible rhaBAD expression system (plasmids pCK351, pCK353, pCK354 and pCK355, which we make available to the community through Addgene, numbers 129696-129699, respectively) represents the best combination of high-level induced expression and undetectable levels of basal (non-induced) expression reported for any inducible system in cyanobacteria to date. As fundamental, general-purpose parts, transcriptional terminators support such different approaches and are equally applicable to improving production of any of the types of native or heterologous products for which cyanobacteria are studied, including biofuels, pharmaceutical compounds, nutrients and pigments.

Supplementary Materials: Supplementary materials can be found at http://www.mdpi.com/2076-2607/7/8/263/s1. Figure S1. Efficiency of terminators in E. coli strains DH10B and MG1655; Figure S2. The effect of terminator insertion upstream of chromosomally-integrated DNA on transcriptional read-through from chromosomal promoters, at 192 h; Figure S3. Growth of Synechocystis cells transformed with terminator plasmids, pAS001-pAS020; Figure S4. Growth of Synechocystis cells transformed with insulated plasmids pCK351, pCK353, pCK354 or pCK355; Table S1. Plasmids and oligonucleotides used in this study. Reference [3] is cited in the Supplementary Materials.

Author Contributions: C.K. and J.H. designed the study; all authors contributed to experimental design; C.K., A.S. and G.T. performed experiments; C.K., G.T. and J.H. prepared the manuscript with input from A.S. and L.D. 
Funding: This work was supported by the Biotechnology and Biological Sciences Research Council [BB/M011321/1 to JTH].

Conflicts of Interest: The authors declare no competing financial interest. The funders had no role in the design of the study; in the collection, analyses, or interpretation of data; in the writing of the manuscript, or in the decision to publish the results.

\section{References}

1. Berla, B.M.; Saha, R.; Immethun, C.M.; Maranas, C.D.; Moon, T.S.; Pakrasi, H.B. Synthetic biology of cyanobacteria: Unique challenges and opportunities. Front. Microbiol. 2013, 4, 246. [CrossRef] [PubMed]

2. Huang, H.-H.; Camsund, D.; Lindblad, P.; Heidorn, T. Design and characterization of molecular tools for a Synthetic Biology approach towards developing cyanobacterial biotechnology. Nucleic Acids Res. 2010, 38, 2577-2593. [CrossRef] [PubMed]

3. Kelly, C.L.; Taylor, G.M.; Hitchcock, A.; Torres-Méndez, A.; Heap, J.T. A Rhamnose-Inducible System for Precise and Temporal Control of Gene Expression in Cyanobacteria. ACS Synth. Biol. 2018, 7, 1056-1066. [CrossRef] [PubMed]

4. Ramey, C.J.; Barón-Sola, Á.; Aucoin, H.R.; Boyle, N.R. Genome Engineering in Cyanobacteria: Where We Are and Where We Need To Go. ACS Synth. Biol. 2015, 4, 1186-1196. [CrossRef] [PubMed]

5. Vijayan, V.; Jain, I.H.; O'Shea, E.K. A high resolution map of a cyanobacterial transcriptome. Genome Biol. 2011, 12, R47. [CrossRef] [PubMed]

6. Rippka, R. Isolation and purification of cyanobacteria. Methods Enzymol. 1988, 167, 3-27. [PubMed]

7. Kelly, C.L.; Liu, Z.; Yoshihara, A.; Jenkinson, S.F.; Wormald, M.R.; Otero, J.; Estévez, A.; Kato, A.; Marqvorsen, M.H.S.; Fleet, G.W.J.; et al. Synthetic Chemical Inducers and Genetic Decoupling Enable Orthogonal Control of the rhaBAD Promoter. ACS Synth. Biol. 2016, 5, 1136-1145. [CrossRef] [PubMed]

8. Chen, Y.-J.; Liu, P.; Nielsen, A.A.K.; Brophy, J.A.N.; Clancy, K.; Peterson, T.; Voigt, C.A. Characterization of 582 natural and synthetic terminators and quantification of their design constraints. Nat. Methods 2013, 10, 659-664. [CrossRef] [PubMed]

9. Geerts, D.; Bovy, A.; de Vrieze, G.; Borrias, M.; Weisbeek, P. Inducible expression of heterologous genes targeted to a chromosomal platform in the cyanobacterium Synechococcus sp. PCC 7942. Microbiology 1995, 141, 831-841. [CrossRef] [PubMed]

10. Miyake, K.; Abe, K.; Ferri, S.; Nakajima, M.; Nakamura, M.; Yoshida, W.; Kojima, K.; Ikebukuro, K.; Sode, K. A green-light inducible lytic system for cyanobacterial cells. Biotechnol. Biofuels 2014, 7, 56. [CrossRef] [PubMed]

11. Jacobsen, J.H.; Frigaard, N.-U. Engineering of photosynthetic mannitol biosynthesis from $\mathrm{CO} 2$ in a cyanobacterium. Metab. Eng. 2014, 21, 60-70. [CrossRef] [PubMed]

12. Jeng, S.T.; Gardner, J.F.; Gumport, R.I. Transcription termination by bacteriophage T7 RNA polymerase at rho-independent terminators. J. Biol. Chem. 1990, 265, 3823-3830. [PubMed]

13. Cambray, G.; Guimaraes, J.C.; Mutalik, V.K.; Lam, C.; Mai, Q.-A.; Thimmaiah, T.; Carothers, J.M.; Arkin, A.P.; Endy, D. Measurement and modeling of intrinsic transcription terminators. Nucleic Acids Res. 2013, 41, 5139-5148. [CrossRef] [PubMed]

14. Liu, D.; Pakrasi, H.B. Exploring native genetic elements as plug-in tools for synthetic biology in the cyanobacterium Synechocystis sp. PCC 6803. Microb. Cell Fact. 2018, 17, 48. [CrossRef] [PubMed]

15. Mitschke, J.; Georg, J.; Scholz, I.; Sharma, C.M.; Dienst, D.; Bantscheff, J.; Voss, B.; Steglich, C.; Wilde, A.; Vogel, J.; et al. An experimentally anchored map of transcriptional start sites in the model cyanobacterium Synechocystis sp. PCC6803. Proc. Natl. Acad. Sci. USA 2011, 108, 2124-2129. [CrossRef] [PubMed]

16. Taylor, G.M.; Mordaka, P.M.; Heap, J.T. Start-Stop Assembly: A functionally scarless DNA assembly system optimized for metabolic engineering. Nucleic Acids Res. 2018. [CrossRef] [PubMed]

(C) 2019 by the authors. Licensee MDPI, Basel, Switzerland. This article is an open access article distributed under the terms and conditions of the Creative Commons Attribution (CC BY) license (http://creativecommons.org/licenses/by/4.0/). 